\title{
Optically Tunable Mesoscale CdSe Morphologies Via INORGANIC PHOTOTROPIC GROWTH
}

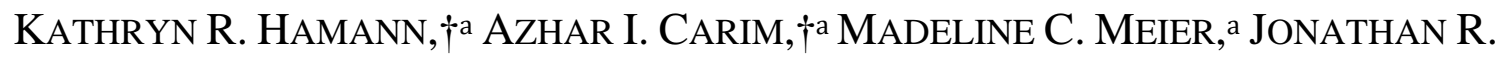

ThOmpson, ${ }^{\mathrm{b}}$ Nicolas A. BATARA, ${ }^{\mathrm{b}}$ IVAN S. Yermolenko, ${ }^{c}$ HARry A. ATWATER ${ }^{\mathrm{b}}$ AND

NATHAN S. LEWIS ${ }^{* a}$

${ }^{a}$ Division of Chemistry and Chemical Engineering, California Institute of Technology, Pasadena, CA 91125,USA.E-mail: nslewis@caltech.edu

${ }^{b}$ Division of Engineering and Applied Sciences, California Institute of Technology, Pasadena, CA 91125, USA

${ }^{c}$ Bruker Corporation, Nano Surfaces Division, San Jose, CA 95134, USA

$\dagger$ These authors contributed equally (KRH and AIC) 


\section{S1. Contents}

This document contains a description of the experimental and modeling/simulation methods utilized in this work (Sections S2 and S3), additional scanning-electron micrographs and two-dimensional Fourier transform data (Section S4), additional computer simulation data (Section S5), and a list of associated references (Section S6). 


\section{S2. Experimental Methods}

Materials and Chemicals $\mathrm{H}_{2} \mathrm{SO}_{4}$ (ACS Reagent, J. T. Baker), buffered HF improved etchant (Transene), In (99.999 \%, Alfa Aesar), Ga (99.999 \%, Alfa Aesar), $\mathrm{SeO}_{2}$ (99.999 \%, Acros Organics), $\mathrm{CdSO}_{4}\left(99+\%\right.$, Sigma-Aldrich), and $\mathrm{CS}_{2}(99.9+\%$, Alfa Aesar) were used as received. $\mathrm{H}_{2} \mathrm{O}$ with a resistivity $\geq 18.2 \mathrm{M} \Omega \mathrm{cm}$ (Barnstead Nanopure System) was used throughout. Aucoated $\mathrm{n}^{+}-\operatorname{Si}(100)(<0.005 \Omega \mathrm{cm}$, As-doped, $525 \pm 25 \mu \mathrm{m}$, single-side polished, Addison Engineering) was used as a substrate for deposition. Flash-Dry Ag Paint (SPI Supplies), EP21ARHTND Epoxy (MasterBond), and nitrocellulose-based nail polish were used to assemble the working electrodes.

Substrate Preparation $\mathrm{n}^{+}-\mathrm{Si}$ wafers were etched with buffered $\mathrm{HF}_{(\mathrm{aq})}$ for $30 \mathrm{~s}$, rinsed with $\mathrm{H}_{2} \mathrm{O}$, dried under a stream of $\mathrm{N}_{2(\mathrm{~g})}$, and then immediately transferred to an electron-beam metal evaporator with a base pressure $<10^{-5}$ torr. Using an accelerating voltage of $10 \mathrm{kV}$, a $10 \mathrm{~nm} \mathrm{Ti}$ adhesion layer was deposited on the polished side of the wafer with a $50 \mathrm{~mA}$ deposition current and a $50 \mathrm{~nm}$ Pt capping layer was then deposited with a $150 \mathrm{~mA}$ current. The wafers were then transferred to a RF sputterer in which $100 \mathrm{~nm}$ of Au was deposited on top of the Pt using a RF power of $80 \mathrm{~W}$. The Au-topped Si sections were cut into square 0.50 by $0.50 \mathrm{~cm}$ sections for use as deposition substrates.

Electrode Preparation One end of a Sn-coated Cu wire (22 AWG) was bent to form a small, flat coil and the wire was threaded through glass tubing $(6 \mathrm{~mm} \mathrm{O}$. D.) such that the coil was just outside the tubing. Epoxy was applied to seal the end of the tube from which the coil protruded. A eutectic mixture of Ga and In was scratched with a carbide-tipped scribe into the unpolished back surfaces of the Au-topped Si sections. The wire coil was then contacted to the unpolished surface and affixed with Ag paint. Nail polish was applied to insulate the unpolished face, the wire-coil 
contact, and the exposed wire between the coil and epoxy seal. Immediately before deposition, the surface of each electrode was briefly cleaned using a stream of $\mathrm{N}_{2}(\mathrm{~g})$.

Electrode Illumination Illumination for photoelectrochemical growth was provided by light-emitting diode (LED) sources (Thorlabs) including narrowband sources with respective

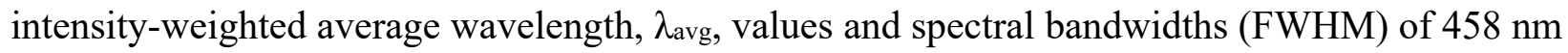
and $20 \mathrm{~nm}$ (SOLIS-460), and $528 \mathrm{~nm}$ and $32 \mathrm{~nm}$ (SOLIS-525B or SOLIS-525C). Additionally, a broadband LED source (SOLIS-3C) was utilized in conjunction with a $550 \mathrm{~nm}$ bandpass filter (Edmund Optics 33-330) to produce a spectral distribution with $\lambda_{\text {avg }}$ and FWHM values of $550 \mathrm{~nm}$ and $93 \mathrm{~nm}$. The output of each LED source was collected, collimated, and condensed using a biconvex lens $(\varnothing 50.8 \mathrm{~mm}, \mathrm{f}=60 \mathrm{~mm})$ in conjunction with two aspheric condenser lenses $(\varnothing 25.4$ $\mathrm{mm}, \mathrm{f}=16 \mathrm{~mm}$; Ø50.8 mm, $\mathrm{f}=32 \mathrm{~mm}$ ). A dichroic film polarizer (Thorlabs LPVISE200-A) was inserted before the final lens to effect linear polarization. For experiments involving simultaneous illumination with two sources, a polka dot beam splitter (Thorlabs BPD508-G) was utilized to combine the outputs; both sources were incident upon the beamsplitter at an angle of $45^{\circ}$ from the surface normal and thus generated coaxial output. For all experiments, a 1500 grit ground-glass (N-BK7) diffuser was placed immediately in front of the photoelectrochemical cell to ensure spatial homogeneity of the illumination.

The light intensity incident on the electrode was measured by placing a calibrated $\mathrm{Si}$ photodiode (Thorlabs FDS100) in the photoelectrochemical cell with electrolyte in place of an electrode assembly, and the steady-state current response of that Si photodiode was measured. Depositions were performed with a total light intensity of $I_{0}=0.250 \mathrm{~W} \mathrm{~cm}^{-2}$ unless otherwise indicated. For experiments involving simultaneous illumination with two orthogonally polarized 
sources, an intensity of $0.7 \cdot I_{0}$ was provided by one source and an intensity of $0.3 \cdot I_{0}$ was provided by the orthogonally polarized source.

Photoelectrochemical Growth Photoelectrochemical growth was performed using a BioLogic SP-200 potentiostat and a single-compartment glass cell with a quartz window. A threeelectrode configuration was utilized with a graphite-rod counter electrode $(99.999 \%$, SigmaAldrich) and a $\mathrm{Ag} / \mathrm{AgCl}$ reference electrode (3.00 M KCl, Bioanalytical Systems). Films were grown from an aqueous solution of $0.00500 \mathrm{M} \mathrm{SeO}_{2}, 0.200 \mathrm{M} \mathrm{CdSO}_{4}$, and $0.100 \mathrm{M} \mathrm{H}_{2} \mathrm{SO}_{4}$. Growth was effected by supplying a current density of $-0.50 \mathrm{~mA} \mathrm{~cm}{ }^{-2}$ at the Au-coated electrode, illuminated as detailed under the above subheading (Electrode Illumination), at room temperature until a charge of $-1.0 \mathrm{C} \mathrm{cm}^{-2}$ had passed. After growth, the electrode was immediately removed from the cell, rinsed with $\mathrm{H}_{2} \mathrm{O}$, and then dried under a stream of $\mathrm{N}_{2}(\mathrm{~g})$. The Au-coated substrate with top-facing Se-Cd film was mechanically separated from the rest of the electrode assembly. The nitrocellulose-based insulation and the majority of the Ag paint and In-Ga eutectic were then removed mechanically.

Chemical Post-Processing Following photoelectrochemical growth as detailed in the above subheading, the substrate and Se-Cd film were transferred to a round-bottom glass flask and immersed in $\mathrm{CS}_{2(1)}$. The $\mathrm{CS}_{2(1)}$ was maintained at the boiling point $\left(46^{\circ} \mathrm{C}\right)$ under reflux for $15 \mathrm{~h}$, to effect the elimination of excess Se from the films and thereby produce stoichiometric CdSe. The electrode was then removed from solution and subsequently rinsed with $\mathrm{H}_{2} \mathrm{O}$ and dried under a stream of $\mathrm{N}_{2(\mathrm{~g}) \text {. }}$

Microscopy Scanning-electron micrographs (SEMs) were obtained with a FEI Nova NanoSEM 450 at an accelerating voltage of $5.00 \mathrm{kV}$ with a working distance of $5 \mathrm{~mm}$ and an inlens secondary electron detector. Scanning-electron micrographs obtained for quantitative analysis 
were acquired with a resolution of 172 pixels $\mu \mathrm{m}^{-1}$ over $\sim 120 \mu \mathrm{m}^{2}$ area whereas micrographs that were used to produce display figures were acquired with a resolution of 344 pixels $\mu \mathrm{m}^{-1}$ over $\sim 2$ $\mu \mathrm{m}^{2}$ areas. Atomic-force micrographs were collected with a Dimension Icon Atomic Force Microscope (Bruker Nano Surfaces) using ScanAsyst-Air probes (Bruker AFM Probes). Micrographs were collected with a resolution of 342 pixels $\mu \mathrm{m}^{-1}$ over $\sim 1 \mu \mathrm{m}^{2}$ areas.

Energy-Dispersive X-ray Spectroscopy Energy dispersive X-ray (EDX) spectroscopy was performed in the SEM using an accelerating voltage of $15.00 \mathrm{kV}$ with a working distance of $5 \mathrm{~mm}$. An Oxford Instruments X-Max Si drift detector was utilized. Spectra were collected in the range of 0 to $10 \mathrm{keV}$ and quantitative film compositions were derived from these spectra using the "INCA" software package (Oxford Instruments).

X-ray Diffraction Grazing incidence X-ray diffraction (GIXRD) was performed using a Bruker D8 Discover diffractometer with a $\mathrm{Cu} \mathrm{K} \alpha$ source and a 2-dimensional Vantec detector. The X-rays were directed at a grazing angle $\omega=0.3^{\circ}$ above the plane of the sample surface and the detector was swept throughout the entire $2 \theta$ range. 


\section{S3. Modeling and Simulation Methods}

Simulation of Film Morphology The growths of the photoelectrochemically deposited films were simulated with an iterative growth model in which electromagnetic simulations were first used to calculate the local photocarrier-generation rates at the film surface. Then, mass addition was simulated via a Monte Carlo method wherein the local photocarrier-generation rate weighted the local rate of mass addition along the film surface.

Growth simulations began with a bare, semi-infinite planar substrate. In the first step, the light-absorption profile under a linearly polarized, plane-wave illumination source was calculated using full-wave finite-difference time-domain (FDTD) simulations ("FDTD Solutions" software package, Lumerical) with periodic boundary conditions along the substrate interface. In the second step, a Monte Carlo simulation was performed in which an amount of mass, equaling that of a 15 $\mathrm{nm}$ planar layer covering the simulation area, was added to the upper surface of the structure with a probability $F$ :

$$
F(G)=G \prod_{i=1}^{3} \frac{x_{i}}{r_{i}} \quad(\text { Equation 1) }
$$

where $G$ is the spatially dependent photocarrier-generation rate at the deposit/solution interface, $x_{i}$ is the fraction of $\mathrm{i}^{\text {th }}$ nearest neighbors occupied in the cubic lattice, and $r_{i}$ is the distance to the $\mathrm{i}^{\text {th }}$ nearest neighbor. The multiplicative sum in the definition of this probability (Equation 1) serves to reduce the surface roughness of the film so as to mimic the experimentally observed surface roughness. A value of $n=1.33$ was used as the refractive index of the growth solution, regardless of wavelength. ${ }^{1}$ Simulations of the film morphology utilized the intensity-weighted average wavelengths, $\lambda_{\text {avg, }}$ of the experimental sources described in Section S2. The electric-field vector of the illumination was oriented parallel to the substrate. A three-dimensional cubic mesh with a lattice constant of $7 \mathrm{~nm}$ was used for the simulations. After the initial Monte Carlo simulation, the 
absorbance of the new, structured film was then calculated in the same manner as for the initial planar film, and an additional Monte Carlo simulation of mass addition was performed. This process of absorbance calculation and mass addition was repeated for a total of 18 iterations.

Dipole Simulations Dipole simulations were performed using a FDTD method. A twodimensional square simulation plane was utilized. Dipoles were arranged with a separation of twice the emission wavelength and the oscillation axis was set perpendicular to the separation axis. 
S4. Additional Scanning-Electron Micrographs and Two-Dimensional Fourier Transform Data

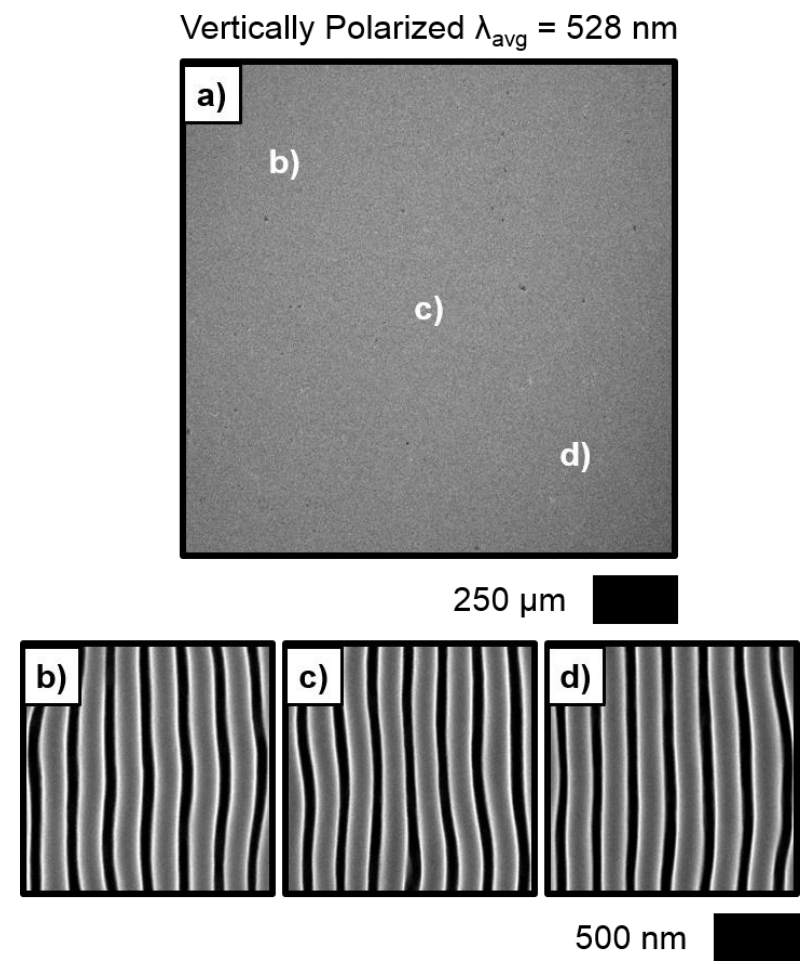

Figure S1. (a) Low-magnification SEM of a film generated using vertically polarized $\lambda_{\text {avg }}=528 \mathrm{~nm}$ illumination. (b)(d) High-magnification SEMs acquired from the areas indicated in the SEM presented in (a). 


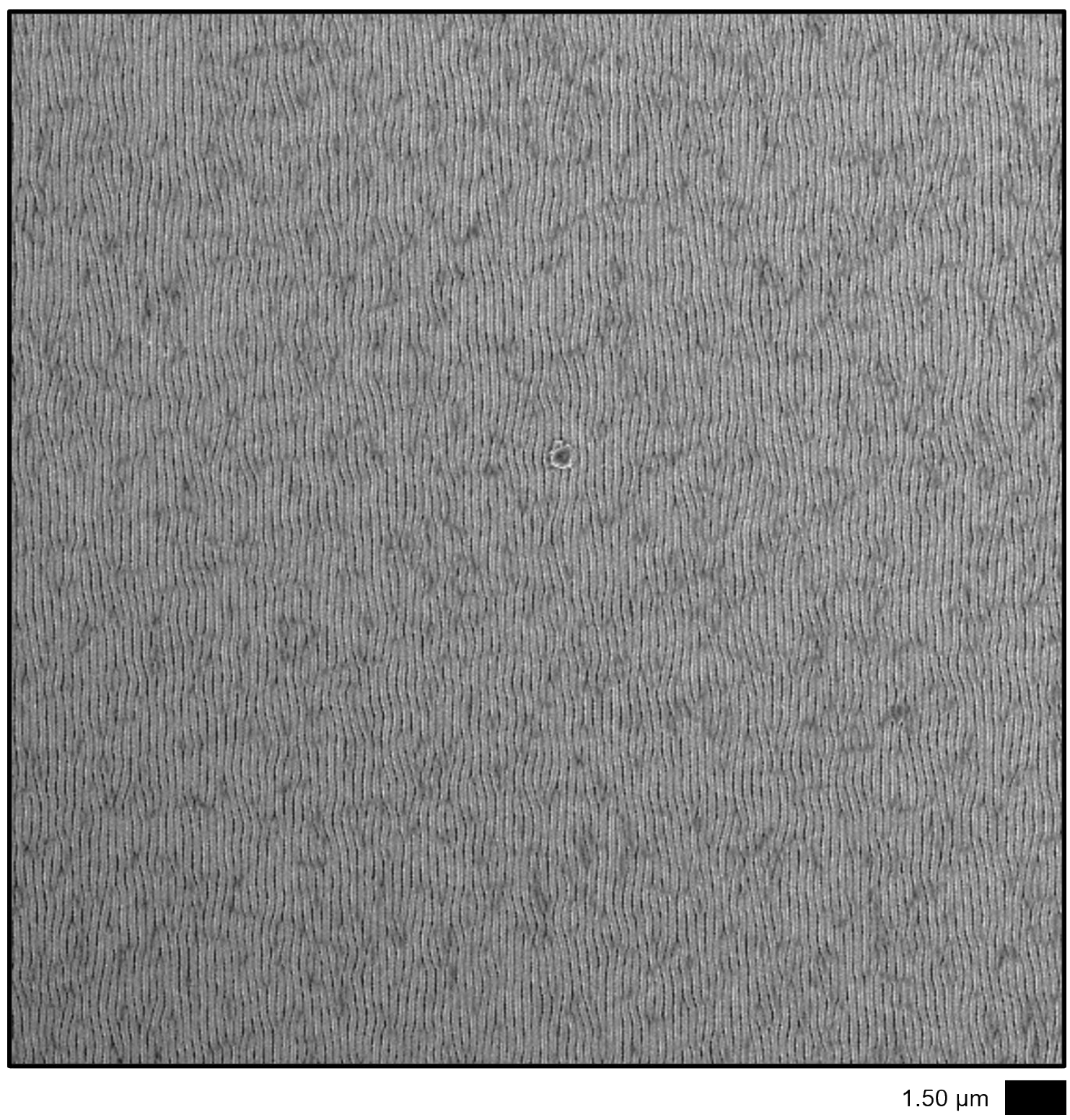

Figure S2. SEM representative of a film generated using vertically polarized $\lambda_{\text {avg }}=528 \mathrm{~nm}$ illumination. 

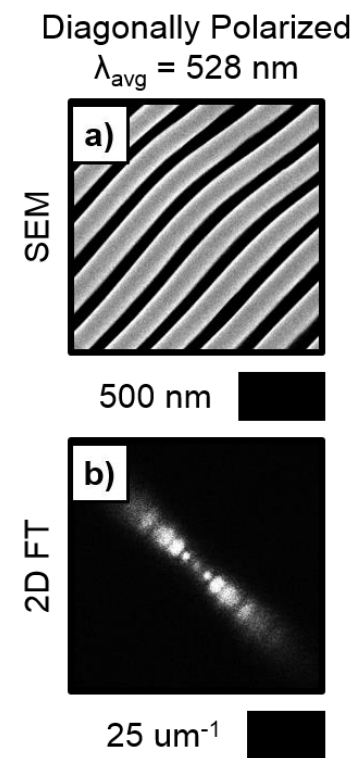

Figure S3. (a) SEM representative of a film generated using diagonally polarized (aligned $\theta=45^{\circ}$ counterclockwise from the horizontal) $\lambda_{\text {avg }}=528 \mathrm{~nm}$ illumination. (b) 2D FT derived from a SEM of the film depicted in (a).

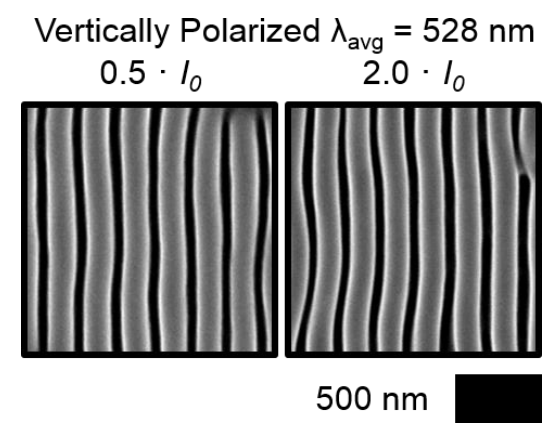

Figure S4. SEMs representative of films generated using vertically polarized $\lambda_{\text {avg }}=528 \mathrm{~nm}$ illumination at the

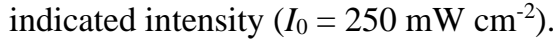




\section{S5. Additional Computer Simulation Data}

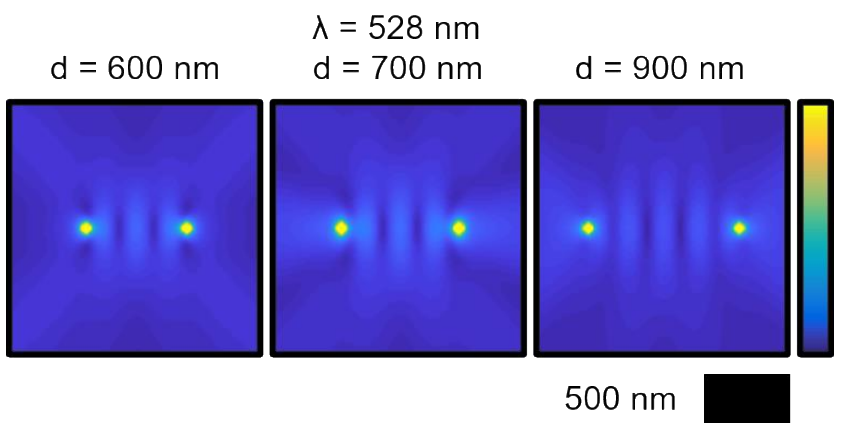

Figure S5. Normalized time-average of the E-field magnitude from two dipoles emitting radiation with a free space wavelength of $\lambda=528 \mathrm{~nm}$ in a medium of refractive index $n=1.33$. Dipoles separated by the indicated distance $\mathrm{d}$ along the horizontal with the oscillation axis perpendicular to the axis of separation.

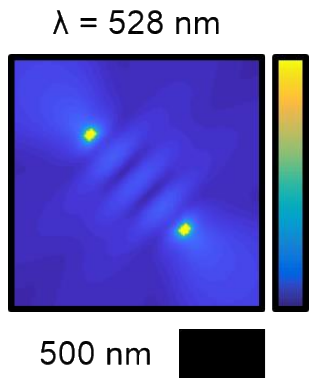

Figure S6. Normalized time-average of the E-field magnitude from two dipoles emitting radiation with a free space wavelength of $\lambda=528 \mathrm{~nm}$ in a medium of refractive index $n=1.33$. Dipoles separated by a distance of twice the wavelength along an axis $\theta=45^{\circ}$ counterclockwise from the horizontal with the oscillation axis perpendicular to the axis of separation.

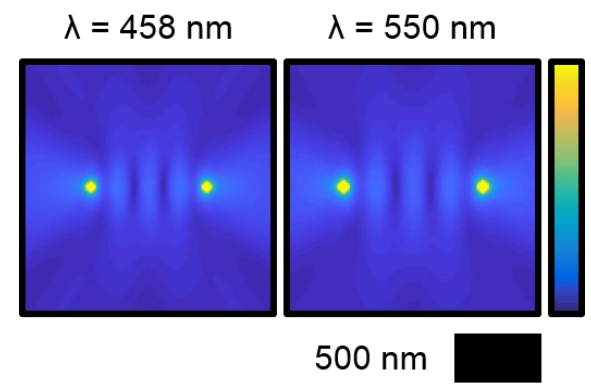

Figure S7. Normalized time-average of the E-field magnitude from two dipoles emitting radiation with the indicated free space wavelength in a medium of refractive index $n=1.33$. Dipoles separated by a distance of twice the wavelength along the horizontal with the oscillation axis perpendicular to the axis of separation. 


\section{S6. References}

1. G. M. Hale and M. R. Querry, Appl. Opt., 1973, 12, 555-563. 